\title{
RF Self-Interference Cancellation for Full-Duplex
}

\author{
B. van Liempd, \\ B. Debaillie, \\ J. Craninckx \\ imec \\ Leuven, Belgium
}

\author{
C. Lavín, \\ C. Palacios \\ TTI \\ Santander, Spain
}

\author{
S. Malotaux, J.R. Long \\ Delft University of \\ Technology \\ Delft, The Netherlands
}

\author{
D.J. van den Broek, \\ E.A.M. Klumperink \\ University of Twente \\ Twente, The Netherlands
}

\begin{abstract}
This paper proposes two RF self-interference cancellation techniques. Their small form-factor enables full-duplex communication links for small-to-medium size portable devices and hence promotes the adoption of full-duplex in mass-market applications and next-generation standards, e.g. IEEE802.11 and 5G. Measured prototype implementations of an electrical balance duplexer and a dual-polarized antenna both achieve $>50 \mathrm{~dB}$ self-interference suppression at $\mathrm{RF}$, operating in the ISM band at $2.45 \mathrm{GHz}$.
\end{abstract}

Keywords-Full-Duplex; RF, Self-Interference Cancellation; Dual-Polarization Antenna; Electrical Balance Duplexer, CMOS

\section{INTRODUCTION}

Full-duplex (FD) communication has the ability to increase data throughput and network efficiency and thus enable sustainable network growth and the introduction of mobile standards such as next-generation IEEE 802.11 and 5G [1]. In contrast with time- and frequency-division duplexing (TDD, FDD), the transmitter (Tx) and receiver (Rx) operate at the same time and channel in an FD communication link (Fig. 1). Hence, self-interference (SI) originating from the Tx degrades the Rx signal-to-noise ratio (SNR) much more compared to conventional (TDD or FDD) links. Large SI at RF can even cause compression at the first receiver input stage, namely the low noise amplifier (LNA), thereby crippling the whole communication link between devices.

Self-interference cancellation (SIC) is crucial for FD and a very high total $\mathrm{SIC}>100 \mathrm{~dB}$ is required, likely requiring multi-stage SIC [2]. Fig. 2 shows an example architecture with multiple SIC stages across the Rx chain, i.e. RF SIC before the LNA, post-mixer analog SIC at baseband frequencies and digital SIC. Sufficient RF SIC is required in order to avoid problematic nonlinearity in the Rx chain such as intermodulation distortion and even clipping. Subsequent cancellation stages must ensure sufficient signal-to-interference ratio (SIR) to establish a robust device-to-device link across operating conditions, including cancellation of (delayed) reflected SI [3],[4].

In recent literature, several designs have demonstrated several SIC techniques [3]-[9]. However, so far, some RF SIC implementations only achieve $<50 \mathrm{~dB}$ SIC [7],[8]. Other solutions [5],[6],[9] use at least two antennas, inappropriate for small form-factor devices due to their required physical spacing. Moreover, using multiple antennas introduces nulls in the antenna beam pattern, degrading coverage. In contrast, single antenna solutions allow integration in compact form-factor devices while ensuring good far-field coverage.

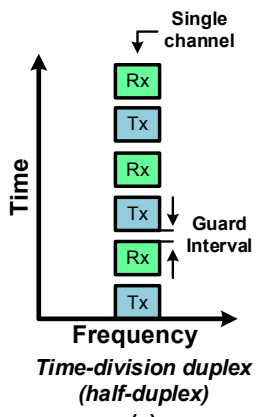

(a)

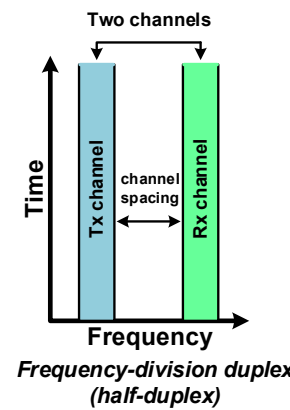

(b)

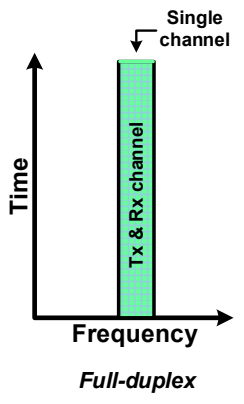

(c)

Figure 1. Half-duplex TDD (a) and FDD (b) compared to FD (c).

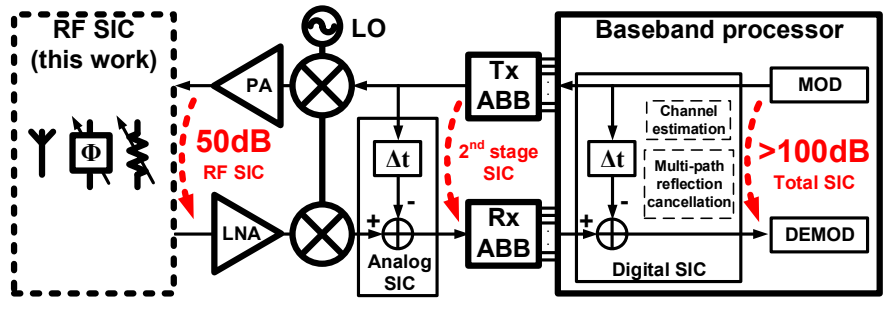

Figure 2. Example of RF, analog and digital SIC applied to a FD transceiver.

In this paper, two single-antenna RF SIC techniques are proposed. First, an electrical balance duplexer provides $>50 \mathrm{~dB}$ SIC across $>6 \mathrm{MHz}$, measured with a real planar inverted-F antenna (PIFA) at $2.45 \mathrm{GHz}$. Second, a dual-feed antenna using dual-polarization achieves $>50 \mathrm{~dB}$ of measured isolation by itself and $>65 \mathrm{~dB}$ across $>10 \mathrm{MHz}$ when a tuned RF SIC loop is operated in conjunction with the antenna element.

These demonstrated results obtain sufficient SIC in an commercially attractive small form-factor, allowing FD to become a mainstream mode of operation for devices, such as tablets, smartphones, and other end-user equipment.

\section{PROPOSED RF SIC TECHNIQUES}

\section{A. RF SIC with an Electrical Balance Duplexer}

In conventional single-antenna devices, surface-acoustic wave (SAW)-based duplexers provide the required isolation between the transmitter and the receiver for standards operating in FDD mode. Furthermore, they provide out-of-band filtering to resolve blocker issues in the $\mathrm{Rx}$ path. However, such duplexers are based on fixed-frequency filters, which only allow antenna sharing when the Tx and Rx operate concurrently on different frequencies. 


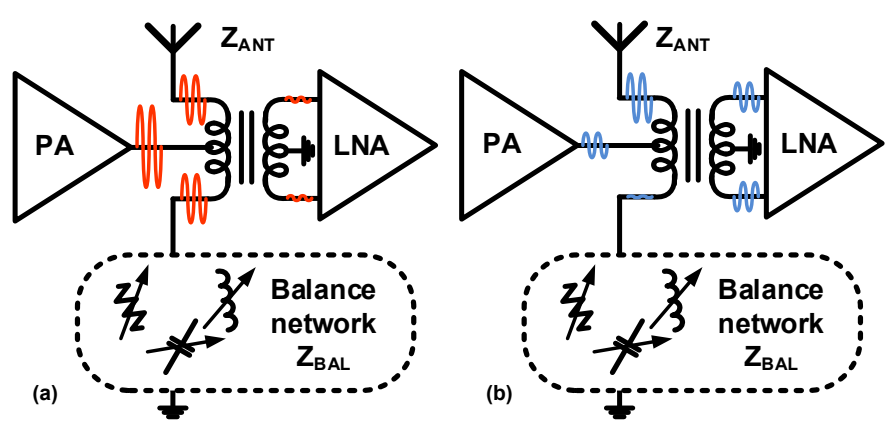

Figure 3. Electrical balance duplexer operating principle for (a) Tx operation and (b) Rx operation: in FD, (a) and (b) occur at the same time and frequency.

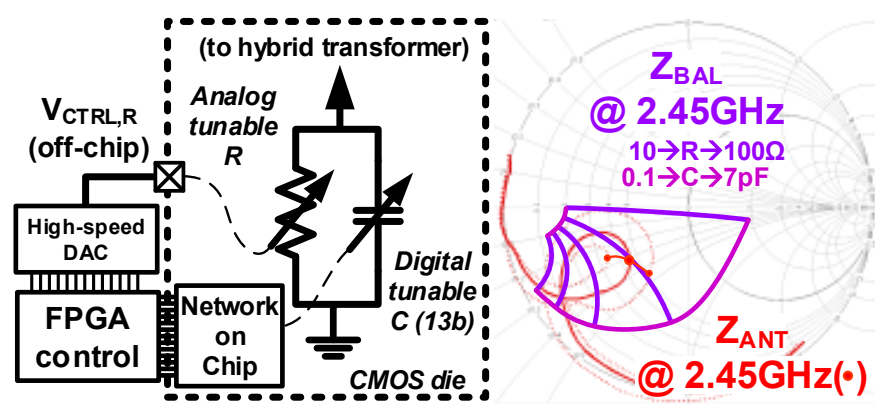

Figure 4. Proposed balance network topology and tuning circuitry (left) and measured impedance variations of $Z_{\mathrm{ANT}}$ (PIFA) with $Z_{\mathrm{BAL}}$ tuning range (right).

For $\mathrm{FD}$ concurrent $\mathrm{Tx}$ and $\mathrm{Rx}$ operation at the same frequency is required. Recently, the use of hybrid transformers to achieve signal cancellation based on electrical balance (EB) has been proposed to achieve tunable duplexer filters for FDD [10]-[12]. In this paper, we apply this technique in the context of full-duplex and show how it provides RF self-interference cancellation for compact radio devices.

Fig. 3 shows the conceptual operation of an EB duplexer, comprising a hybrid transformer and so-called balance network, which is essentially a tunable dummy load impedance. Ideally, the PA signal splits up exactly between the antenna and the balance network, so that no differential voltage excitation occurs and the hybrid transformer essentially subtracts the voltage across these impedances. The limit to the SIC provided by this electrical balance condition is limited by the accuracy with which the balance network can mimic $\mathrm{Z}_{\mathrm{ANT}}$ [10]. By tuning the resistance and reactance of $Z_{\mathrm{BAL}}$ independently with high precision, $>50 \mathrm{~dB}$ of SIC can be achieved across the channel bandwidth (BW). In virtue of this purely passive cancellation process, any noise and nonlinearity products generated in the transmitter are also cancelled by this subtraction.

The principle of electrical balance is applicable to both FDD and FD: the Tx-to- $Z_{\mathrm{ANT}}$ path as well as the $Z_{\mathrm{ANT}}$-to-Rx path have a wideband transformer response, while $\mathrm{Tx}$-to-Rx cancellation is required at both the $\mathrm{Tx}$ and $\mathrm{Rx}$ frequencies simultaneously (FDD) [12] or instead at a single frequency only (FD), a grace of FD operation.

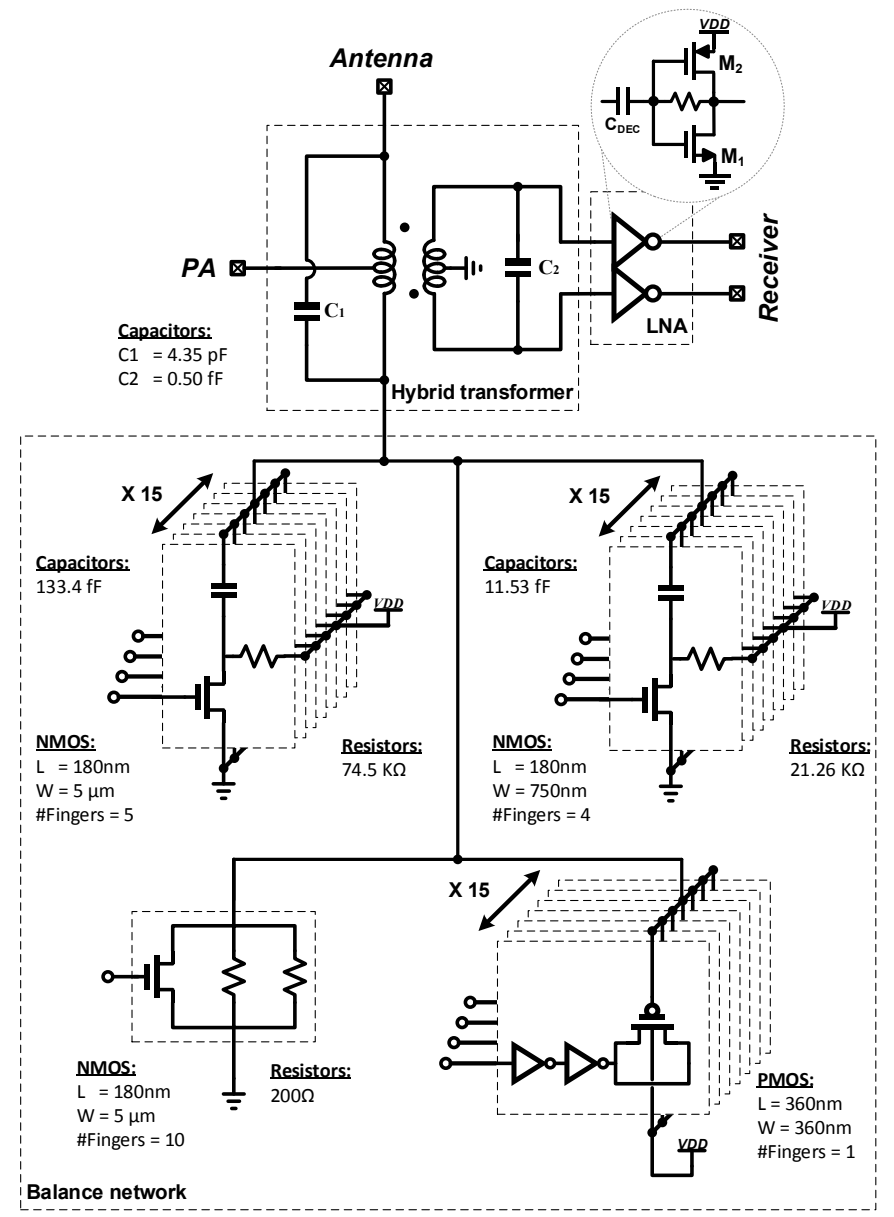

Figure 5. Schematic of the FD electrical balance duplexer circuit.

The on-chip impedance tuning range must cover the frequency-dependent impedance of a real antenna, which varies across environmental conditions. The non-ideal antenna interconnect causes an additional impedance shift that also must be taken into account during the design of $Z_{\mathrm{BAL}}$. For the measured PIFA impedance (Fig. 4), a parallel resistor and capacitor mimic the required impedance range that covers the variations of $Z_{\mathrm{ANT}}$ including the antenna interconnect.

Fig. 5 shows the circuit schematic of the implemented duplexer. The balance network shows three binary tunable capacitor banks (coarse, fine and superfine), the latter of which is using PMOS transistors as tuned capacitors to achieve the required minimum capacitance-step. The resistor is implemented using an NMOS transistor and a parallel resistance, achieving an analog tuning range from 10-100 $\Omega$.

Note that the Tx power splits across $Z_{\mathrm{ANT}}$ and $Z_{\mathrm{BAL}}$, hence a $>3 \mathrm{~dB}$ insertion loss is expected excluding transformer loss. Compared to current SAW-based FDD solutions with $\sim 2.5 \mathrm{~dB}$ Tx path loss, the penalty is rather small to enable small form-factor FD. Finally, the EB duplexer topology provides a wideband transfer and hence no filtering in the Rx path. For this reason, either blocker-resilient SDR architectures [10] or additional filters are required for proper operation. 


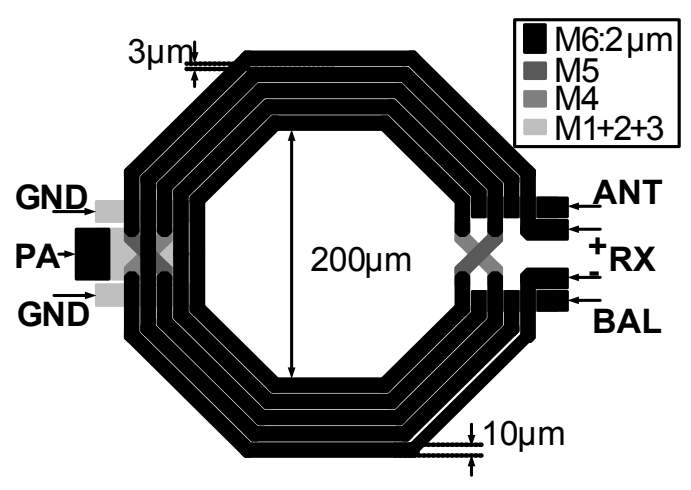

Figure 6. Hybrid transformer layout.

The LNA is a pseudo-differential inverter in feedback configuration, compatible with SDR receivers such as [13]. Since no standing waves caused by reflections can occur in the short interconnect between LNA and transformer, no $50 \Omega$ interface is required. Therefore, in this design, the LNA is noise-matched using a high $10 \mathrm{k} \Omega$ feedback resistance only for DC-coupling. Noise matching lowers the LNA noise contribution compared to a conjugate match [11], so that FD sensitivity can be competitive to FDD solutions, given enough total SIC. This also implies that no power is absorbed by the LNA. From the antenna point of view, instead, the PA and balance network impedances offer a real impedance of about $25 \Omega$, so that the antenna-port $\mathrm{S} 11$ is reasonable ( $\operatorname{sim} .-8 \mathrm{~dB}$ ) and $>80 \%$ incident power is absorbed: the transformer senses the voltage across the antenna and couples it into the LNA. The total noise factor F, including the transformer losses and LNA, is given by [11]:

$\mathrm{F}=1+\mathrm{r}+\frac{\mathrm{R}_{\mathrm{ANT}}}{\mathrm{R}_{\mathrm{p}}}(1+\mathrm{r})^{2}+\frac{2 \gamma}{\mathrm{g}_{\mathrm{M}} \mathrm{R}_{\mathrm{ANT}}} \frac{\mathrm{L}_{1}}{\mathrm{k}^{2} \mathrm{~L}_{2}}\left[1+\frac{\mathrm{R}_{\mathrm{ANT}}}{\mathrm{R}_{\mathrm{p}}}(1+\mathrm{r})\right]^{2}$

where skewing factor $r=1, \mathrm{R}_{\mathrm{ANT}}=50 \Omega$ (assuming a perfect antenna), total parallel transformer resistance $\mathrm{R}_{\mathrm{p}}=50.75 \Omega$, LNA transconductance $\mathrm{g}_{\mathrm{M}}=38 \mathrm{mS}$ and excess noise factor $\gamma=2 / 3$ give a theoretical $8.9 \mathrm{~dB}$ cascaded Rx NF at $2.45 \mathrm{GHz}$, mostly limited by the transformer losses. This cascaded NF is acceptable, since it is expected that despite our best-efforts to achieve high SIC, residual self-interference will limit the full-duplex link budget, not thermal noise [2].

Fig. 6 shows the layout of the hybrid transformer used in this design. It is an interwound, octogonal design, with a grounded secondary winding center-tap, using the primary winding center-tap for injection of the PA signal. The primary winding self-inductance is $0.5 \mathrm{nH}$ with a peak $\mathrm{Q}$ of 10 and the secondary winding self-inductance is $3 \mathrm{nH}$ with a peak $\mathrm{Q}$ of 10.6 , while the coupling factor $k$ between the two windings is 0.74 (optimized for Q-factor using EMX simulations). The self-inductance ratio follows the physical winding ratio $\mathrm{N}$ of 1:3. Capacitors $\mathrm{C}_{1}$ and $\mathrm{C}_{2}$ set the self-resonance to $2.45 \mathrm{GHz}$, together with the input impedance of the LNA, which is capacitive in nature due to the gate oxide capacitance.

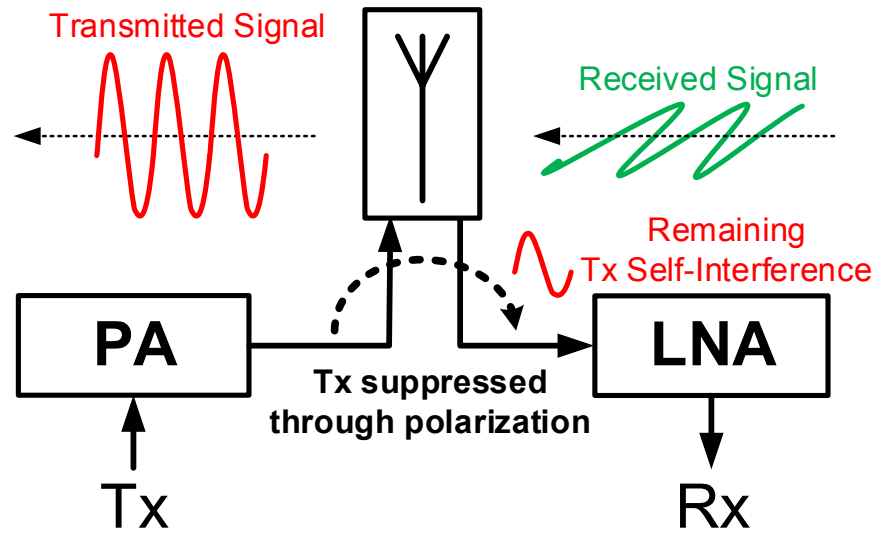

Figure 7. Operating principle of dual-polarized antenna in full-duplex scenarios.

\section{B. RF SIC with a Dual-Polarized Dual-Feed Antenna}

This section describes the second RF SIC approach, a compact single antenna design which uses dual-polarization to achieve high self-interference suppression.

The polarization of an antenna describes the orientation and sense of the electric field vector radiated by the antenna. The maximum energy transferred between two electromagnetic waves will only occur if both waves have the same orientation and sense of electric field vector. On the contrary, if both polarizations are different, there will be a reduction in energy transfer due to a polarization mismatch. Ideally, the transferred energy between orthogonal polarizations is zero [14]. Considering this fact, FD systems can use antennas with orthogonal polarizations to minimize the transferred energy from the transmitter to the receiver.

This idea of using orthogonal polarizations in order to prevent self-interference has been used in recent FD work. In [15], orthogonal polarizations for $\mathrm{Tx}$ and $\mathrm{Rx}$ signals are employed, but using two antennas separated $35 \mathrm{~cm}$. Alternatively, this paper proposes a solution that uses only one antenna element to generate both orthogonal polarizations in the same radiating aperture. For that reason, the proposed dual-polarized antenna is suitable for compact form-factor devices, e.g. laptops or tablets.

In the case of full-duplex systems, the two antenna feed ports are connected to the Tx and Rx, respectively, as shown in Fig. 7. The isolation between antenna feed-points will determine the remaining Tx signal level that leaks into the Rx.

Microstrip patch antennas are widely used as dual-polarized elements in wireless communication systems due to their low profile, low cost and easy integration with RF circuits. The main concern for dual-polarization operation is to achieve high isolation and low cross-polarization level simultaneously. This is difficult, especially for compact antenna solutions, where both excitation ports are close and the coupling between them is high. 


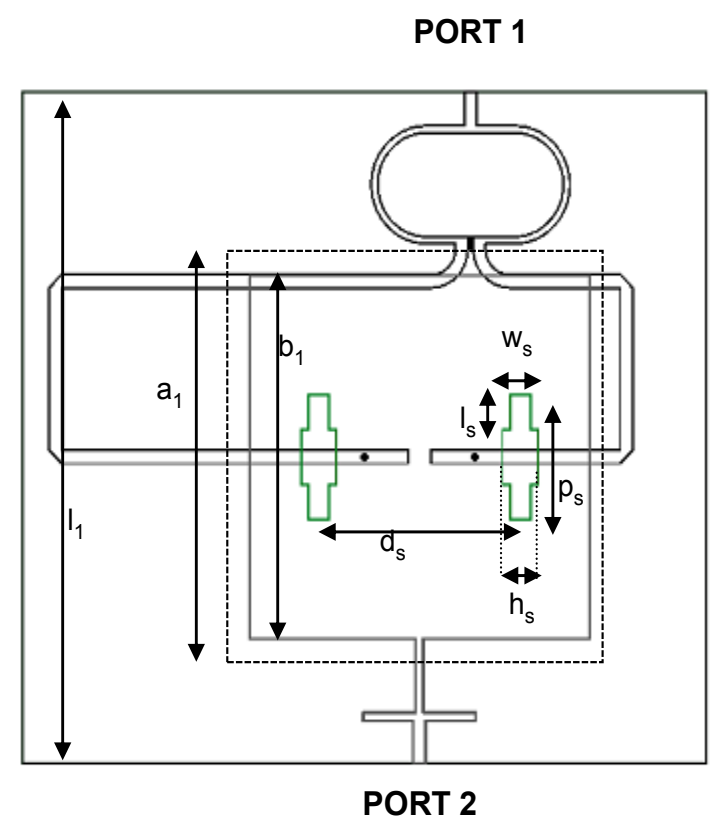

(a)

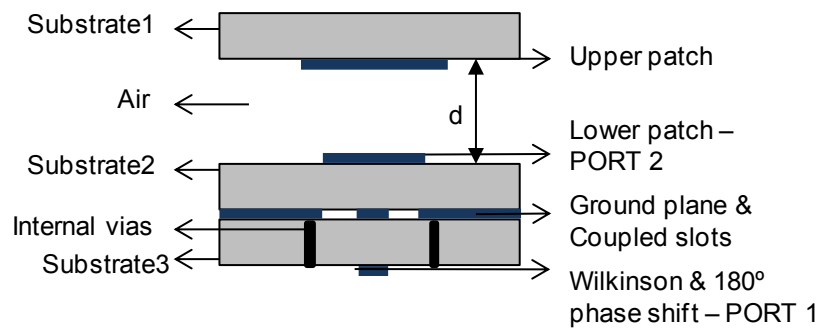

(b)

Figure 8. Structure of the dual-polarized micostrip antenna. (a) Top view. (b) Multilayer antenna stack-up.

A variety of feeding techniques to achieve good isolation and low cross-polarization level have been published in literature. Classical approaches use common orthogonal offset slots [16], however this geometry only provides $18 \mathrm{~dB}$ of isolation over the antenna impedance bandwidth. H-shaped slots can also be used to increase the isolation, reducing the coupling between excitation ports. In [17], two orthogonal H-shaped slots with more than $36 \mathrm{~dB}$ of isolation are proposed. Other feeding techniques propose L-shaped feeds [18], or hybrid excitation combining in-phase aperture-coupled feeds and out-of-phase gap-coupled probe feeds [19], achieving up to $40 \mathrm{~dB}$ isolation over the antenna impedance bandwidth.

In this work, the geometry of the antenna is based on two stacked patches that are excited via a slot-coupled feed and microstrip line, targeting $>50 \mathrm{~dB}$ isolation. The geometry size is limited by this requirement. Fig. 8 shows the proposed dualpolarized patch antenna geometry. The antenna consists of three dielectric substrate layers and one air layer separating the two square patches by a distance $d$. All substrate layers have the same thickness and dielectric constant. The upper patch is placed on the bottom of substrate 1 and the lower patch is placed on top of substrate 2 . The lower patch is excited by means of a microstrip

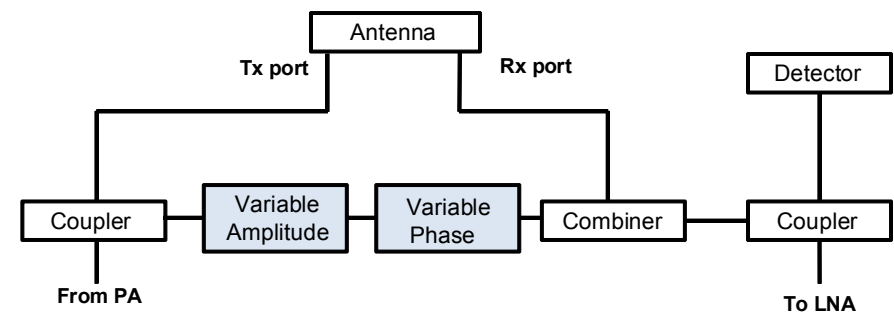

Figure 9. Block diagrama of the tunable analog cancellation network combined with dual-polarized antenna.

TABLE I. ANTENNA DESIGN PARAMETERS.

\begin{tabular}{|c||c|}
\hline $\mathrm{d}[\mathrm{mm}]$ & 10.98 \\
\hline $\mathrm{l}_{1}[\mathrm{~mm}]$ & 90.00 \\
\hline $\mathrm{a}_{1}[\mathrm{~mm}]$ & 32.59 \\
\hline $\mathrm{b}_{1}[\mathrm{~mm}]$ & 30.45 \\
\hline $\mathrm{ds}[\mathrm{mm}]$ & 20.61 \\
\hline $\mathrm{ps}[\mathrm{mm}]$ & 12.93 \\
\hline $\mathrm{ls}[\mathrm{mm}]$ & 4.74 \\
\hline $\mathrm{hs}[\mathrm{mm}]$ & 2.39 \\
\hline ws $[\mathrm{mm}]$ & 3.01 \\
\hline
\end{tabular}

line with matching stubs for $50 \Omega$ impedance matching at Port 2. Substrates 2 and 3 are separated by an internal ground plane with two slots. These two coupled slots are excited by means of two feed lines which are printed on the back of substrate 3 . These lines have a $180^{\circ}$ phase shift between them and are combined/divided by a Wilkinson divider. This provides the Port 1 feeding network. Generation of higher-order propagation modes are avoided by exciting the patch from two opposite points with $180^{\circ}$ phase shift. This improves the polarization purity and the patch isolation. Moreover, a pair of internal vias improves the radiation characteristics of the overall antenna. Using this antenna geometry, Port 1 and Port 2 excite linear polarizations in the vertical and horizontal planes, respectively.

The antenna structure shown in Fig. 8 has been optimized for $2.45 \mathrm{GHz}$ using a Rogers substrate with a 3.55 dielectric constant and a $0.508 \mathrm{~mm}$ thickness. Final design parameters for the antenna are summarized in Table 1.

Considering this antenna in a full-duplex scenario, near-by objects close to the antenna can cause degradation of the antenna isolation. Moreover, reflections from external objects can rotate the Tx polarisation, generating signals with the same $\mathrm{Rx}$ polarisation. These signals will leak into the $\mathrm{Rx}$, causing self-interference. Taking the degradation of interference suppression at antenna level into account, it is useful to complement the antenna solution with a tunable cancellation stage in order to improve the total RF SIC.

Fig. 9 illustrates the proposed tunable cancellation circuitry in combination with the dual-port antenna. A copy of the Tx RF signal (including its in-band Tx impairments) is attenuated, phase-shifted and combined with the RF received signal before the LNA. The attenuator and phase shifter are tuned to compensate the self-interference leakage due to imperfect isolation between $\mathrm{Tx}$ and $\mathrm{Rx}$, and maintain $\mathrm{a}>50 \mathrm{~dB}$ total SIC at RF. The coupler/combiner have $0.2 / 0.7 \mathrm{~dB}$ loss, respectively. 


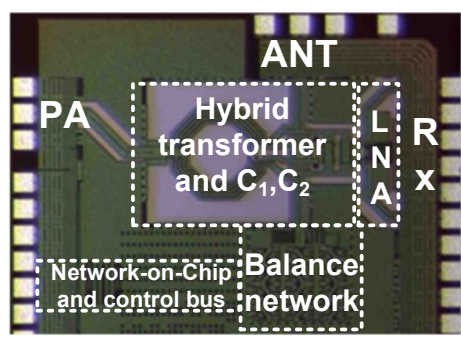

(a)

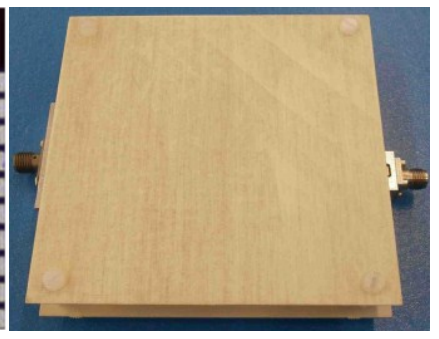

(b)
Figure 10. (a) Duplexer chip and (b) dual-feed, dual-polarization antenna.

\section{MEASUREMENT RESULTS}

\section{A. EB Duplexer RF SIC Measurement Results}

Fig. 10(a) shows a photograph of the $0.4 \mathrm{~mm}^{2}$ EB-duplexer chip fabricated in a bulk $0.18 \mu \mathrm{m}$ CMOS process. An off-chip DAC is used to set $\mathrm{V}_{\mathrm{CTRL}}$ for the on-chip resistor tuning. The PIFA used in conjunction with the prototype (not shown) operates at $2.45 \mathrm{GHz}$, and measures $26 \times 5 \mathrm{~mm}$.

Fig. 11(a) shows the measured $Z_{\mathrm{BAL}}$ range for which $>60 \mathrm{~dB}$ Tx-to-Rx isolation is observed when sweeping the impedance at the antenna port using a Maury MT982EU impedance tuner. The worst-case impedance variations of the PIFA due to environmental condition changes are covered by the tuning range of $Z_{\mathrm{BAL}}$ at $2.45 \mathrm{GHz}$, both in simulation and measurement.

Fig. 11(b) shows the measured and simulated isolation curves at $2.45 \mathrm{GHz}$ when the PIFA is connected to the duplexer, after tuning $Z_{\mathrm{BAL}}$ to achieve an electrical balance condition. A bandwidth of $6 \mathrm{MHz}$ is measured for $>50 \mathrm{~dB}$ SIC, which fits the simulation results.

Fig. 11(c) shows total gain and cascaded NF from antenna to LNA output. As the impedance balance changes, so does the gain and NF (the 'Max. R' setting reflects the EB condition). At resonance $(2.15 \mathrm{GHz})$, measured $\mathrm{NF}$ is $9.4 \mathrm{~dB}$, slightly higher than calculated from (1), which holds at resonance. At $2.45 \mathrm{GHz}, \mathrm{NF}$ is about $7 \mathrm{~dB}$ in balance condition. Measured/simulated Tx insertion loss is $3.4 \mathrm{~dB}$ at resonance.

\section{B. Antenna-based RF SIC Measurement Results}

Fig. 10(b) depicts the dual polarized antenna. The measured return loss and self-interference suppression (S21) are shown in Fig. 12(a) and Fig. 12(b), respectively. Good agreement between simulated and measured results is obtained, except for a displacement in Port1 matching. However, the return loss of the antenna is still $<-10 \mathrm{~dB}$ across $10 \mathrm{MHz} \mathrm{BW}$.

The performance of the active cancellation network has also been experimentally evaluated, using a HMC624LP4 attenuator and a HMC928LP5E analog phase shifter. Both were manually tuned to optimize the total RF SIC. Fig. 12(b) shows $>65 \mathrm{~dB}$ total RF SIC over $10 \mathrm{MHz}$ BW when the active cancellation network is used with the dual-polarized antenna, improving upon the isolation achieved by the antenna with $15 \mathrm{~dB}$.
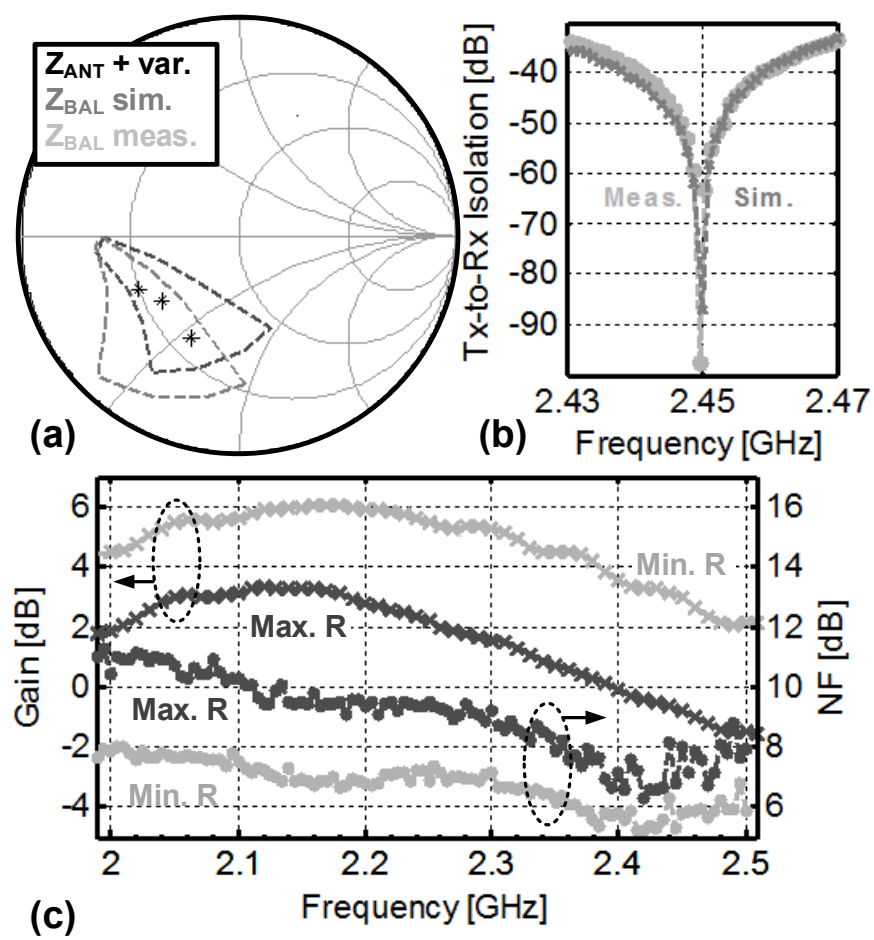

Figure 11. FD duplexer measurements: (a) $\mathrm{Z}_{\mathrm{BAL}}$ tuning range at $2.45 \mathrm{GHz}$, (b) SIC at $2.45 \mathrm{GHz}$ with PIFA and (c) Rx-path gain and NF versus frequency.

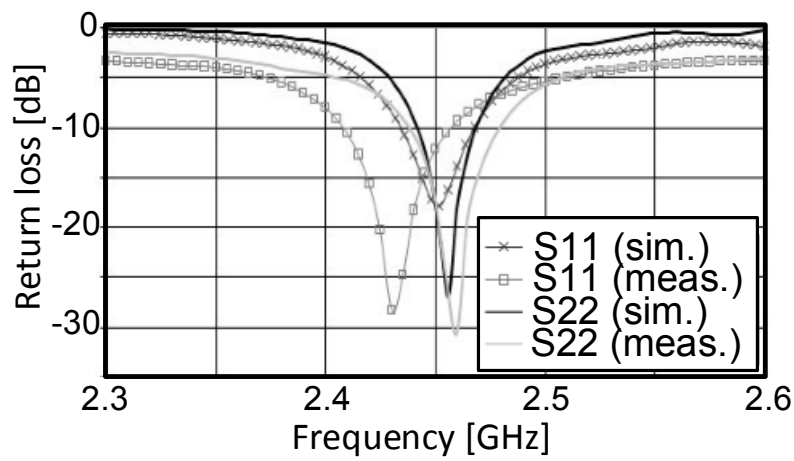

(a)

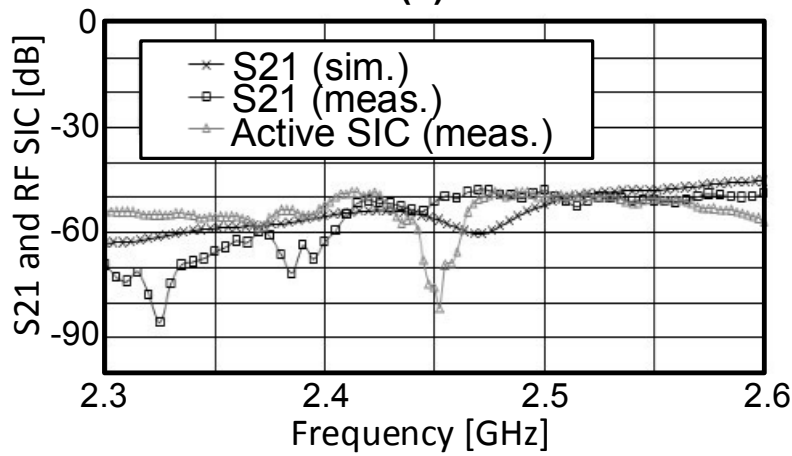

(b)

Figure 12. Measured and simulated S11 (a) and S21 (b) for the dual-polarized antenna and measured active cancellation. 


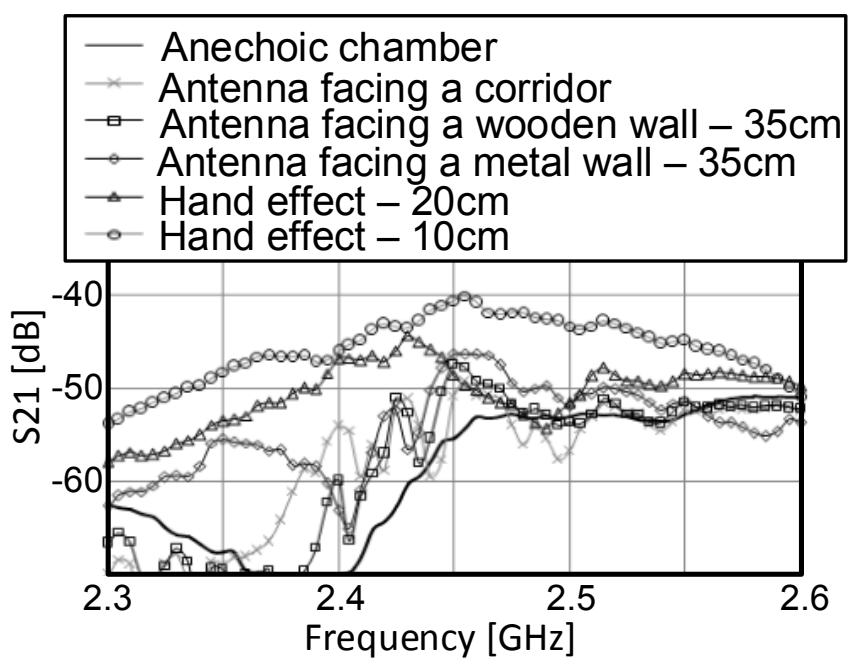

Figure 13. Effect of environmental changes on the antenna SI-suppression.

The performance of the antenna in terms of isolation has been evaluated considering different external objects around the antenna, as shown in Fig. 13. Measured results show how the isolation between $\mathrm{Tx}$ and $\mathrm{Rx}$ ports is $>45 \mathrm{~dB}$ considering different objects around the antenna at a distance up to $20 \mathrm{~cm}$. Only when the antenna is completely blocked at $10 \mathrm{~cm}$ from the antenna, the isolation between ports worsens to $>40 \mathrm{~dB}$. The cancellation loop compensates for this degradation by $>15 \mathrm{~dB}$.

All measurements were done with at least $0 \mathrm{dBm}$ Tx power.

\section{CONCLUSION}

This work demonstrates two RF self-interference cancellation techniques that achieve $>50 \mathrm{~dB}$ cancellation for the direct Tx-to-Rx leakage path, directly at RF.

On one hand, an electrical balance duplexer is proposed to achieve a tunable SIC characteristic with $6 \mathrm{MHz}$ bandwidth, covering the antenna impedance variations of a real antenna in typical operating conditions. It operates concurrently in time and frequency domain, with a $\mathrm{Tx}$ insertion loss of $3.4 \mathrm{~dB}$ and an cascaded Rx NF of $7 \mathrm{~dB}$. The electrical balance duplexer prototype including the antenna occupy just $130 \mathrm{~mm}^{2}$, demonstrating the feasibility of full-duplex at a small footprint.

On the other hand, a dual-polarized antenna is proposed to achieve $>50 \mathrm{~dB}$ isolation between its 2 ports. The antenna solution is complemented by an active cancellation network making the solution robust against environmental effects. Using this network, $15 \mathrm{~dB}$ isolation improvement is achieved for a $10 \mathrm{MHz}$ bandwidth. The antenna size of $9 \times 9 \times 1.2 \mathrm{~cm}$ is compatible with small devices such as laptops and routers.

This work brings full-duplex transceivers a step closer to implementation. Future work includes combining the presented RF SIC techniques with additional cancellation using analog techniques (post-mixer) and digital techniques for cancellation of multi-path reflections, in conjunction with accurate channel estimation. Also, the presented EB duplexer only supports up to $0 \mathrm{dBm} T \mathrm{Tx}$ signals, and further work is needed to improve the power-handling capability. For both techniques, MIMO compatibility still needs to be demonstrated. Finally, real-time on-line automated tuning of any cancellation algorithm is a challenging digital processing task, which will be tackled in future work.

\section{ACKNOWLEDGMENT}

The authors would like to thank H. Suys and L. Pauwels (imec) for assistance during measurement and Integrand Software for EMX.

\section{REFERENCES}

[1] DUPLO consortium, "DUPLO FP7 D1.1: System scenarios and technical requirements for full-duplex concept", April 30, 2013 On-line: http://fp7-duplo.eu/images/docs/Deliverables/D1_1_v_1.0.pdf

[2] B. Debaillie et al. "Analog/RF Solutions Enabling Compact Full-Duplex Wireless Communication Radios," IEEE Journal on Selected Areas in Communications (JSAC), accepted for publication, 2014.

[3] D. Bharadia et al.,"Full duplex radios" ACM Proc. of the SIGCOMM Conference (SIGCOMM'13), pp.375-386, 2013.

[4] Y. Choi and H. Shirani-Mehr, "Simultaneous Transmission and Reception: Algorithms, Design and System Level Performance," IEEE Trans. Wireless Commun., vol. 12, no. 12, Dec. 2013.

[5] M. Duarte et al., "Design and Characterization of a Full-duplex Multiantenna System for WiFi networks,"IEEE Trans. On Vehicular Technology, No.99, 2013.

[6] J. Choi et al., "Achieving Single Channel, Full Duplex Wireless Communication," Int. Conf. on Mob. Comp. and Netw., Sept. 2010.

[7] M. E. Knox, "Single Antenna Full Duplex Communications using a Common Carrier," in Proc. 13th Annual IEEE Wireless and Microwave Technology Conference, Apr. 2012, pp. 1-6.

[8] C. Cox and E. Ackerman, "Demonstration of a Single-Aperture, Full-Duplex Communication System," in Proc. Radio and Wireless Symposium, 2013, pp. 148-150.

[9] A. K. Khandani, "Methods for Spatial Multiplexing of Wireless Two-Way Channels," United States Patent, Oct. 2010.

[10] M. Mikhemar et al., "A Multiband RF Antenna Duplexer on CMOS: Design and Performance," IEEE J. Solid-State Circuits, Vol.48, No.9, pp.2067-2077, Sept. 2013.

[11] S.H. Abdelhalem et al., "Hybrid Transformer-Based Tunable Differential Duplexer in a 90-nm CMOS Process," IEEE Trans. Microw. Theory and Techniques, Vol.61, No.3, pp.1316-1326, March 2013.

[12] S.H. Abdelhalem et al., "Hybrid transformer-based tunable integrated duplexer with antenna impedance tracking loop," IEEE Custom Integ. Circ. Conf. (CICC), Sept. 2013.

[13] B. van Liempd et al., "IIP2 and HR Calibration for an 8 Phase Harmonic Recombination Receiver in 28nm," IEEE Custom Integ. Circ. Conf. (CICC), Sept. 2013.

[14] Cushcraft Corporation, "Antenna Polarization Considerations in Wireless Communications Systems", 1999-2002.

[15] Evan Everett et al., " Passive Self-Interference for Full-Duplex Infrastructure Nodes", IEEE Trans. Wireless Communications, Vol. 13, No.2, Feb. 2014.

[16] A. Adrian and D.H. Schaubert, "Dual aperture-coupled microstrip antenna for dual or circular polarization", Electron. Lett., Vol.23, No.23, Nov. 1987.

[17] S.-C. Gao et al., "Dual-polarized slot-coupled planar antenna with wide bandwidth", IEEE Trans. Antennas Propag., Vol.51, No.3, Mar. 2003.

[18] H. Wong et al., "Design of dual-polarized L-probe patch antenna arrays with high isolations," IEEE Trans. Ant. Prop., 2004.

[19] T.-W. Chiou et al., "Broad-band dual-polarized single microstrip patch antenna with high isolation and low cross polarization", IEEE Trans. Antennas Propag., Vol.50, No. 3, Mar 2002. 